\section{Variable Pressure Scanning Electron Microscopy For Nonconductive and Volatile Samples}

Larry D. Hanke, P.E

Materials Evaluation and Engineering, Inc., Plymouth, MN

During the past two decades, the scanning electron microscope (SEM) has become an indispensable tool for morphological observation in materials and biological sciences. With energy dispersive x-ray spectroscopy (EDS) and wavelength dispersive spectroscopy (WDS), rapid chemical characterization of microscopic features has also become commonplace. As developments in manufacturing methods allow fabrication of ever smaller devices and quality control has achieved higher priority, demand for microscopic characterization by SEM has continuously increased.

Many applications where SEM/EDS evaluation could be useful involve samples that are not electrically conductive. Nonconductive samples are subject to a buildup of electrons on the examined surface. This buildup of electrons, or "charging" eventually causes scattering of the incoming electron beam, which interferes with imaging and analysis. Nonconductive samples have traditionally required pretreatment, by coating with a conductive film, before SEM examination.

Samples that contain substantial water or other materials that volatilize in high vacuum also present challenges for SEM examination. These samples require controlled drying to allow the SEM chamber to reach high vacuum and to prevent deformation of the sample at the SEM vacuum.

In many cases, sample pretreatment is only partially effective in preparing the samples for SEM examination. For example, wet samples change character when dried, thus appearing different in the SEM examination than in their native state. For nonconductive, porous samples, it is difficult to get the conductive coating into the subsurface spaces. Charge buildup at the pores continues to interfere with the SEM examination.

In addition, coating confuses analysis by the EDS and WDS techniques. Carbon is the typical material for coating when microanalysis is required. Early EDS detectors could not detect carbon. Therefore, carbon coating was not a severe limitation for microanalysis. Today's EDS systems detect carbon and lighter elements, as light as beryllium. Carbon applied to sample surfaces obviously will interfere with detection of carbon in the sample and can inhibit detection of other elements present.

As the SEM is increasingly used for routine evaluations, there is increasing demand for examination without pretreatment. Coating is not an alternative for in-process inspection of components to be put into service where the coating affects the components' suitability for service. Coating may also be unacceptable for preparation of samples that should not have their character altered, such as historic materials or evidence in legal actions.

SEM examination can be performed at low accelerating voltages to diminish the effects of charging. Field emission SEMs are especially effective for imaging at sufficient low accelerating voltages to prevent charging. $X$-ray analysis, however, is not possible at these low accelerating voltages. Minimum voltages of 5 to $10 \mathrm{KV}$ are required for reliable x-ray analysis, which is not sufficiently low to prevent charging on many materials. In addition, field

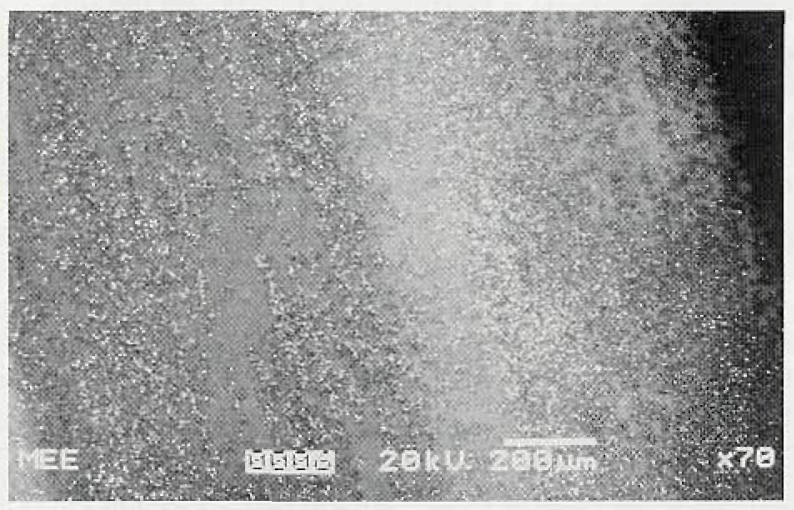

Figure 1: Natural rubber coated with silicone lubricant. emission SEMs are considerably more expensive than instruments with the common tungsten-filament sources.

An answer to the problems of charging and volatile samples is the new scanning electron microscopes that operate without exposing the sample to high vacuum. These microscopes are referred to alternately as environmental, lowvacuum, high-pressure, or variable-pressure SEMs. Higher pressures in the SEM sample chamber offer two primary benefits compared to traditional highvacuum chambers. First, a higher pressure minimizes the outgassing from volatile samples. Secondly, by allowing a controlled amount of gas into the chamber, charging is diminished on nonconductive samples.

The advantages of a higher pressure in the sample chamber are obvious for wet and volatile samples. The higher pressure decreases the rate of volatilization or outgassing. This decreases the drying and deformation of wet samples. Since the sample chamber can tolerate higher pressures, any outgassing does not inhibit operation of the microscope. Figure 1 is a micrograph of natural rubber with a coating of silicone lubricant. Direct SEM examination shows the lubricant distribution on the rubber surface.

For nonconductive samples, the advantage of higher pressure is less obvious. When gas molecules in the sample chamber are struck by the electron beam, the gas is ionized. These positive ions are attracted to and neutralize the negative charge building up on the nonconductive specimens. By controlling the pressure in the sample chamber, the number of gas molecules intercepting the electron beam is maintained at a level that is sufficient to prevent charging, but does not deflect the beam sufficiently to prevent imaging and microanalysis.

At the higher pressures, accelerating voltages up to the maximum capacity of the SEM (typically $30 \mathrm{KV}$ for high-performance SEMs) can be used for imaging and microanalysis of nonconductive samples. Figures 2,3 , and 4 show micrographs of nonconductive materials. No pretreatment, which could interfere with imaging and analysis, is required for examination of these specimens.

Within the past 2 years, higher pressure operating capabilities have been made available on moderately-priced, easy-to-operate, high-performance SEMs. The best of these microscopes are excellent conventional high-vacuum SEMs, with high-resolution secondary electron imaging, as well as the capability to operate at variable pressure. These SEMs have all of the features that provide the versatility, usability, and cost-effectiveness on which so many SEM users have come to depend. In addition, there is no adaptation necessary to equip these instruments for microanalysis.

The new generation of variable-pressure scanning electron microscopes has brought the higher-pressure capability out of the research laboratories and into the quality assurance and failure analysis laboratories in manufacturing companies and service organizations. This development will continue increased demand for scanning electron microscopy in an ever widening range of applications.

The variety of materials and components that has been studied in our laboratory using the variable-pressure SEM include: lubricated metals and polymers (Figure 1), biological samples, cloth (Figure 2), polymer films, plastic components (Figure 3), printed-circuit boards (Figure 4), ceramics, painted and coated metals, explanted medical devices with attached tissue, metal components encapsulated in polymer, and corroded, contaminated parts. In many of these cases, evaporative or sputter coating was either undesirable or not possible. As we gain more experience with this new technique, additional applications will be found where SEM was once not an alternative.

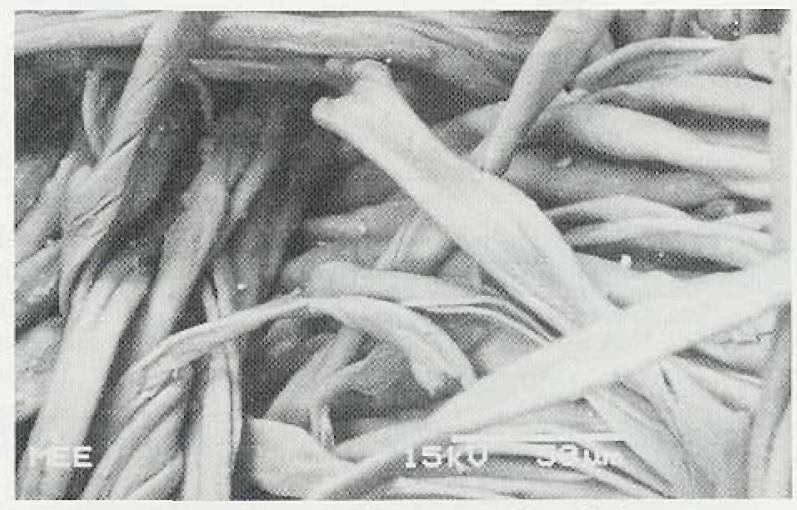

Figure 2: Woven cotton-fiber cloth. 